\title{
Skilled memory in expert figure skaters
}

\author{
JANICE M. DEAKIN \\ Queen's University, Kingston, Ontario, Canada \\ and \\ FRAN ALLARD \\ University of Waterloo, Waterloo, Ontario, Canada
}

\begin{abstract}
The present studies extend skilled-memory theory to a domain involving the performance of motor sequences. Skilled figure skaters were better able than their less skilled counterparts to perform short skating sequences that were choreographed, rather than randomly constructed. Expert skaters encoded sequences for performance very differently from the way in which they encoded sequences that were verbally presented for verbal recall. Tasks interpolated between sequence and recall showed no significant influence on recall accuracy, implicating long-term memory in skating memory. There was little evidence for the use of retrieval structures when skaters learned the brief sequences used throughout these studies. Finally, expert skaters were able to judge the similarity of two skating elements faster than less skilled skaters, indicating a faster access to semantic memory for experts. The data indicate that skaters show many of the same skilled-memory characteristics as have been described in other skill domains involving memorization, such as digit span and memory for dinner orders.
\end{abstract}

It has become common to study the nature of expertise by attempting to determine how experts in a particular skill domain differ from their less skilled counterparts. Such studies have shown experts to be superior for memory tasks involving typical instances of information from their particular skill domains (e.g., Chi, Glaser, \& Farr, 1988 ). This memory superiority is interpreted as reflecting the size of the skill-specific semantic memory base of the expert, essentially making a memory task into a pattern-recognition task for the expert.

Many of the skill domains studied to date have required expertise in problem solving - for example, the games of chess (Chase \& Simon, 1973a, 1973b), bridge (Charness, 1979), and Go (Reitman, 1976), medical diagnosis (e.g., Groen \& Patel, 1988), solving physics problems (Chi, Feltovich, \& Glaser, 1981), and computer programming (e.g., Soloway, Adelson, \& Ehrlich, 1988). The most recent work has evaluated skilled memory directly by analyzing situations in which the recall of large amounts of information is the skill (Ericsson, Chase, \& Faloon, 1980; Ericsson \& Polson, 1988). These studies of skilled memorizers have led to a theoretical account of the skill of the expert known as skilled-memory theory (Chase \& Ericsson, 1982).

Skilled-memory theory is a framework that has the following five characteristics (Ericsson \& Polson, 1988). First, experts encode information using existing semanticmemory patterns. Second, information is stored via

Correspondence should be addressed to Janice M. Deakin, School of Physical and Health Education, Queen's University, Kingston, Ontario K71 3N6, Canada. retrieval structures. The cues are utilized as mnemonic aids while encoding to allow for rapid access upon recall. Third, information is encoded into long-term memory and consequently may be recalled for long periods of time. Fourth, speed of encoding improves with practice. Fifth, as implied by the first principle, memory skill is specific to the domain in which it was acquired initially. Support for these characteristics comes from studies of the development of digit-span skill (Chase \& Ericsson, 1982), observations of a skilled waiter (Ericsson \& Polson, 1988), and investigations of mental calculators (Staszewski, 1988).

In this paper, we apply skilled-memory theory to a motor-skill domain, the domain of figure skating. Previous investigations of motor skills from an expert perspective have shown that basketball and field hockey players are similar to chess experts in their ability to recall large amounts of meaningful game information after a 5-sec exposure to a slide (Allard, Graham, \& Paarsalu, 1980; Starkes \& Deakin, 1984). However, basketball and field hockey involve aspects of problem solving in determining offensive and defensive strategies, much like in chess. Thus, it is not surprising that the players of these sports show cognitive skills similar to those of experts in other domains. In contrast, another category of sport skill exists for which strategy and problem solving are not important factors for success. For such skills as gymnastics, dance, diving, and figure skating, the athlete must perform very complex movements in a specific pattern.

The emphasis for these skills is on consistency and accuracy of output rather than on the selection of an appropriate strategy. The skills also involve a memory component, in that gymnasts and figure skaters must recall large numbers of individual items (movements, in this 
case) comprising a routine or program, as do the memoryskill experts in the digit-span and restaurant-order work. The purpose of the present paper is to determine whether experts from this secondary category of sport skill (i.e., figure-skating experts) show the same skilled-memory characteristics as do digit-span experts and expert waiters.

Starkes, Deakin, Lindley, and Crisp (1987) have previously studied memory for sequences of choreographed and randomly selected ballet movements in skilled and unskilled children. Skilled dancers were significantly better than less skilled dancers for the recall of choreographed sequences; the skilled dancers were aided by the presence of music at encoding and recall. In the first experiment of the present article, we determined whether or not skilled figure skaters are similar to the young ballet dancers studied by Starkes et al. That is, are skilled skaters more accurate in their recall of skating sequences than less skilled skaters, and does the nature of the sequence (i.e., choreographed or randomly selected) influence their recall? Thus, in Experiment 1, we determined whether or not a domain-specific encoding advantage exists for skilled figure skaters, thus validating the fifth principle of skilled memory as earlier described.

\section{EXPERIMENT 1}

In Experiment 1, expert and novice ice dancers were presented with sequences of eight footwork elements. The sequences were constructed from a common pool of 20 elements, with the constraint that all elements in the pool be in the repertoire of the novice skaters. Structured sequences were choreographed by a club professional, as would be done for a program. Unstructured sequences were constructed by drawing elements randomly from the pool. The sequences were presented on videotape to the subjects, who were required to recall the sequences by performing all the elements they could recall. Because expert and novice skaters differ greatly in their ability to perform the sequences, it could be argued that differences in recall would be due to greater output-capacity demand for the novice skaters. To eliminate this possibility as much as possible, subjects responded by performing the sequences on only half of the trials and naming the elements in a sequence on the other half trials.

\section{Method}

Subjects. The subjects consisted of 10 female figure skaters $(6$ skilled and 4 novice skaters). The skilled skaters were senior silver and gold test skaters; the novices were preliminary and junior bronze test skaters. Each of these test levels requires the execution of specific figure-skating elements. As the test level increases from the preliminary level through to the gold level, the complexities of required skills also increase. The skilled skaters were older (mean age $=16$ years) than the novice skaters (mean age $=14.5$ years).

Apparatus. The stimuli consisted of a videotape of a gold test skater performing 10 structured and 10 random footwork sequences of eight elements each. All elements were chosen from a common pool of 20 elements, with the elements selected being commonly found in the required dance routines of elementary skaters. The structured sequences were choreographed by a club professional, with the constraint that similar combinations of elements were not repeated across trials. One characteristic of choreographed sequences is that foot and body symmetry and cadence were maintained in the transition between elements. The random sequences were random selections from the pool of 20 elements.

Procedure. The sequences were presented on a Sony Betamax video recorder and a Sharp Linytron color monitor. The presentation of choreographed and random sequences was blocked, with viewing order counterbalanced across subjects. Recall conditions were also blocked for recall mode (skating or verbal reporting), with report order again being counterbalanced over subjects. Thus, the subjects performed a block of five trials for each experimental condition.

All testing was done next to the ice surface, with the subjects viewing each sequence twice before recall. The delay between the end of the sequence and the initiation of recall was the same for skating and verbal recall. Each subject's skating recall was recorded on videotape for scoring; verbal recall was recorded by the experimenter.

The subjects were instructed to recall as many elements as they could in the same order as the model. For verbal recall, the subjects were instructed to recall the names of the steps in the same order as that viewed in presentation. The subjects were given as much time as needed to complete recall. All testing was completed in one session of approximately $35 \mathrm{~min}$.

\section{Results}

Recall data were analyzed by an analysis of variance (ANOVA), with group (skilled, novice), recall mode (skating, verbal report), sequence (choreographed, random), and serial position (1-8) as factors. The ANOVA showed a significant main effect for sequence $[F(1,8)=$ $10.8, M S_{\mathrm{e}}=1.67, p=.01$, with choreographed sequences $(M=4.0)$ being recalled with greater accuracy than random sequences $(M=3.5)$. As well, the group $\times$ sequence interaction was significant $[F(1,8)=7.03$, $\left.M S_{\mathrm{e}}=1.67, p=.02\right]$, showing skilled subjects $(M=$ $4.4)$ to be more accurate than novice skaters $(M=3.6)$ for structured sequences. This is the typical group $X$ material interaction found over and over again in the expertise literature. As would be expected, there was a highly significant effect of serial position $[F(7,56)=$ $\left.72.42, M S_{\mathrm{e}}=6.67, p<.001\right]$.

Finally, a significant group $\times$ recall mode $\times$ serial position interaction was found $\left[F(7,56)=2.19, M S_{e}=\right.$ $4.34, p=.049$ ]. This interaction is shown in Figure 1 . It occurred primarily because the skilled subjects showed better recall than did the unskilled subjects in the second half of the verbal-recall sequences. Note that the threeway interaction did not involve the nature of the sequence, choreographed or random; thus, the skilled subjects were better at verbal recall over the second half of the sequence for both types of material. This could have been due to the skilled subjects' greater familiarity with the verbal labels for the skill elements viewed, a possibility that will be explored further in Experiment 2. This interaction is also important because it shows that the expert subjects' better recall was not due to output factors. It could be argued that it required less capacity for the expert to execute the skating elements, leaving spare capacity to rehearse the remaining elements. Were this the case, the 

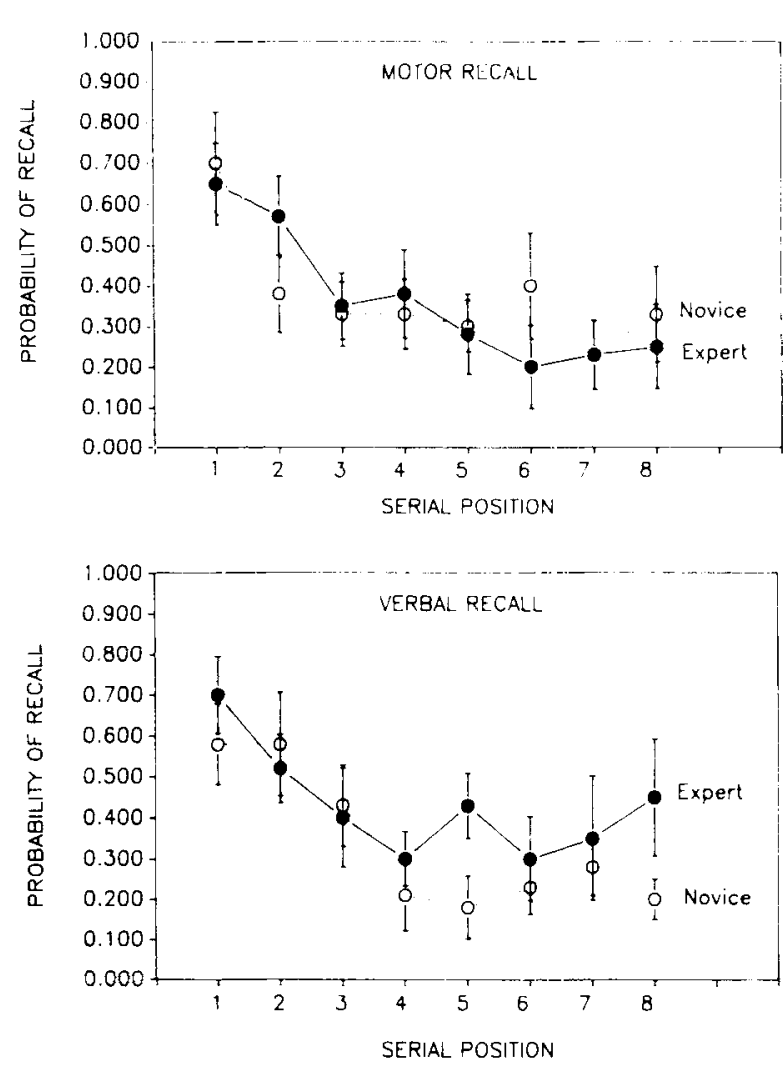

Figure 1. Skill level $\times$ serial position $\times$ modality of recall interaction for Experiment 1.

novice subjects should have shown better recall when naming, rather than performing, the sequences, which is the opposite of what was observed.

\section{Discussion}

The results of Experiment 1 show that the skilled figure skaters had better accuracy of recall for choreographed skating sequences than did the novice skaters. The superior performance for the skilled skaters was observed regardless of how the subjects responded, by skating or verbally reporting their recall. It appears that figure skating is an appropriate domain for the investigation of skilled memory.

One surprising finding of Experiment 1 was the rather low accuracy level of the skaters (approximately 39\% for novice skaters and $\mathbf{4 2 \%}$ for the expert skaters). Although each sequence was comprised of only eight skating elements, each element must be encoded for performance according to the side of the body (left or right), direction of movement (forward or backward), the blade edge (inside or outside), the number of rotations, as well as for the actual name of the element (axel or spin). Therefore, it would take four words to encode each element verbally, for a total of 32 words per sequence. If the skaters were encoding the sequences strictly verbally, novices would be remembering 12.2 words per sequence on average and experts would be remembering 13.4 words per sequence on average. Given the finding that the method of recall (verbal or performing) made no difference to accuracy of performance, the skaters might well have been using words to recall the sequences; this possibility was investigated in Experiment 2.

\section{EXPERIMENT 2}

In Experiment 2, we examined the role of semantic encoding in skaters' recall of skating sequences. One very important aspect of becoming skilled in such activities as figure skating, ballet, or gymnastics is to learn the vocabulary of the particular domain. All of the elements have their names and descriptors; performers must learn the names of, as well as how to perform, the various elements for effective communication between teacher or coach and pupil. Expert skaters might simply have been better able to deal with the semantic encoding of presented lists in Experiment 1. To evaluate this possibility, expert and novice skaters were presented with verbal lists of skating terms for verbal recall. As in Experiment 1, the lists comprised choreographed or random combinations of skating elements. If expert skaters are superior semantic encoders, they should maintain their superior performance over novice skaters for this purely semantic task.

\section{Method}

Subjects. The subjects consisted of 8 skaters -4 expert skaters, who were members of the Canadian National Figure Skating Team, and 4 novices, who were senior bronze or junior silver test free skaters.

Procedure. The skaters were presented with five structured and five unstructured footwork sequences for recall. The names of the elements in each sequence were written on a $3 \times 5$ card. The sequences were identical to those performed by the model in Experiment 1. Each element is made up of both the name of the element and descriptors for side of body, blade edge, and the direction of the movement, for an average of four words per item.

The sequence cards were presented to the skater one at a time, with a viewing time of $4 \mathrm{sec}$ per card, paralleling the presentation time per item in Experiment 1. The subjects recalled by repeating the items in the order of presentation. The presentation of structured and unstructured lists was blocked, with starting sequence counterbalanced across subjects.

\section{Results and Discussion}

Recalls were scored for serial-position accuracy and were analyzed by group (expert, novice), information (structured, random), and serial position (1-8).

The ANOVA showed no significant effect for group or for structure. As would be expected, a main effect was found for serial position $\left[F(7,42)=7.65, M S_{\mathrm{e}}=6.08\right.$, $p<.001]$, with accuracy for Position 8 being better than Positions 4, 5, and 6. Strangely, as shown in Figure 2, a significant interaction between information and serial position emerged $\left[F(7,42)=4.2, M S_{\mathrm{e}}=1.77, p=.001\right]$. The interaction resulted from superior accuracy occurring in the unstructured sequences for Positions 3 and 4 , followed by an increase in accuracy for Positions 7 and 8 for structured lists. A crossover occurred in that Positions 3 and 4 of the unstructured lists were recalled reliably more often than were those positions in the struc- 


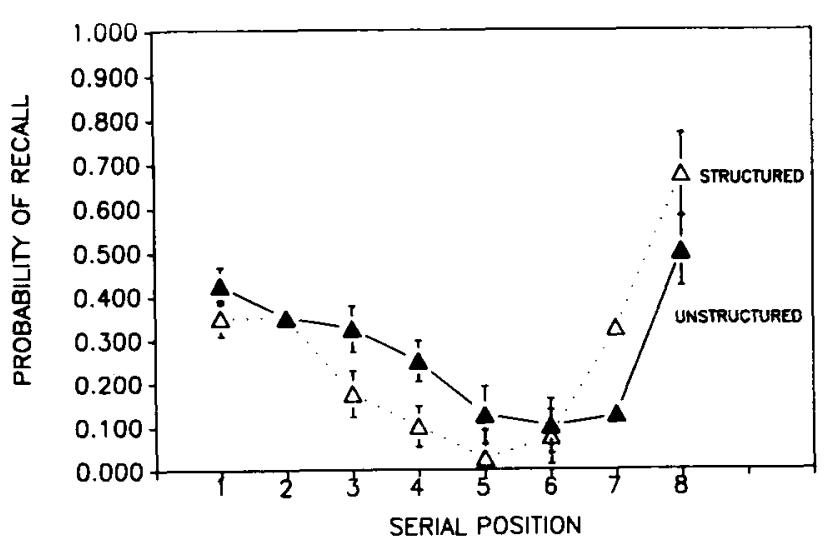

Figure 2. Serial-position curve for Experiment 2.

tured lists, whereas Position 7 of the structured lists displayed significantly higher recall performance than did the same position in the unstructured lists. The theoretical significance of this interaction is unclear. Similar interactions were not apparent in any of the other experiments discussed in this paper in which subjects were required to translate the form of the presented information to a different modality for recall.

The skill by structure interaction shown in Experiment 1 when skaters had to encode from a model performing skating sequences was not observed for Experiment 2 when skaters encoded the verbal labels of skating terms. One conclusion that could be reached from this observation is that skill differences are a function of labeling the action with the appropriate verbal code, because once the verbal encoding is provided for the skater, skill differences go away. However, such an argument does not take into account other differences between the two studies, in particular the form of the serial-position recall-accuracy data. For Experiment 1, serial-position accuracy shows only primacy, reflecting the skaters' strategy of piling one element onto the next in order. For Experiment 2, serialposition accuracy shows both primacy and recency, as would be found for recall of a typical verbal list (e.g., Zacks, Hasher, Alba, Sanft, \& Rose, 1984). Thus, skaters apparently do not differ as a function of skill level for verbal recall of verbal lists, because they are treating the lists differently from the way in which they would treat actual skating sequences that they would be required to perform. Thus, the encoding strategies that skaters use to learn performance sequences seem to differ from more "general purpose" list-learning strategies.

\section{EXPERIMENT 3}

Thus far, it has been established that skating experts use domain-specific structural knowledge to encode sequences of elements and that the encoding done is not equivalent to verbal list learning. In Experiment 3, we attempt to determine whether skating experts use longterm memory for their encoding, as do the other skilledmemory experts investigated to date. The use of long-term memory in other experts has been most often determined by incidental recall for sequences learned following the experimental session (Chase \& Ericsson, 1982; Ericsson \& Polson, 1988; Staszewski, 1988). Given the busy schedule of national team skaters and the expense of renting ice for testing, incidental recall proved to be impractical for our purposes.

Instead, a technique was used that Charness (1976) employed previously to establish that chess experts utilize long-term memory to hold chess patterns after a brief presentation. Charness simply introduced interpolated activities between the initial presentation of a chess position and subjects' recall. Were chess information stored in limited capacity short-term memory, interpolated activity would reduce recall performance. Charness reported that the information recalled following a 5-sec look at a chess position remained at the same level in spite of interpolated activity, evidence that short-term memory was not being used for the holding of chess information. It should be pointed out that the interpolated tasks used by Charness did produce decrements in performance when subjects attempted to recall consonant trigrams rather than chess positions. The lack of interpolation effect was confirmed by Frey and Adesman (1976), who showed that counting backwards by sevens did not influence the recall of chess diagrams.

In Experiment 3, skaters were asked to perform skating sequences, which they initially observed on cards, following a variety of interpolated tasks. If the skaters were storing sequences in short-term memory, interpolated tasks should result in a reduction in the number of elements recalled, as compared with unfilled interval performance.

Since expert-novice differences were shown in Experiment 1 when a model presented the sequences but were not evident in the sequential presentation of verbal labels in Experiment 2, part of the experts' superiority may be in going from seeing the element to naming it. The use of a visual presentation of the verbal labels in Experiment 3 eliminated this explanation for superior performance, thus leaving it clear that experts excel either in the encoding of the presented elements or in translating the information from encoding to performance. The requirement to perform the sequences in Experiment 3 eliminated the opportunity for the subjects to treat the items as simple word lists.

\section{Method}

Subjects. The subjects consisted of 10 skaters -5 expert skaters ( 3 males, 2 females), who were members of the Canadian National Figure Skating Team (mean age $=17$ years), and 5 senior bronze test club skaters ( 5 females, mean age $=15$ years).

Materials. The stimuli consisted of 10 choreographed free-skating sequences, each eight elements in length. The sequences were created from a pool of 40 items, all within the performance range of both groups of skaters. Each sequence was written on a $5 \times 8$ in. card. The 10 sequences were divided into two series of five sequences each, with the order of sequences within each series being fixed for a particular recall condition.

Procedure. Each subject was tested individually. Prior to testing, a list of the 40 elements used in the study was shown to each subject in order to establish the identity of each individual item. The subjects were tested in four recall conditions: immediate recall, 
Table 1

Proportion Correct Recall as a Function of Interpolated Task

\begin{tabular}{lcccc}
\hline & \multicolumn{4}{c}{ Interpolated Task } \\
\cline { 2 - 5 } Skater & $\begin{array}{c}\text { Immediate } \\
\text { Recall }\end{array}$ & $\begin{array}{c}\text { Unfilled } \\
\text { Delay }\end{array}$ & $\begin{array}{c}\text { Count } \\
\text { Backwards }\end{array}$ & $\begin{array}{c}\text { Skate Around } \\
\text { Perimeter }\end{array}$ \\
\hline Expert & .57 & .66 & .67 & .58 \\
Novice & .48 & .50 & .42 & .44 \\
\hline
\end{tabular}

recall following a $30-\mathrm{sec}$ unfilled delay, recall following $30 \mathrm{sec}$ of counting backward by threes, and recall following $30 \mathrm{sec}$ of skating around the perimeter of the ice.

Recall conditions were blocked and counterbalanced across subjects. Five sequences were presented for each recall condition such that each series of sequences was used the same number of times for each recall condition. It should be pointed out that each subject was exposed to each of the sequences twice in the course of this experiment.

The subjects were informed of the motor-recall condition and instructed to reproduce each sequence in the order presented on the card. Each card was viewed by the subject for $30 \mathrm{sec}$ ( $4 \mathrm{sec}$ per element). All recall performances were videotaped for subsequent analysis.

\section{Results and Discussion}

Items recalled correctly were analyzed by group (expert, novice), serial position (M1-M8), and recall condition (immediate, 30 unfilled, 30 count, 30 skate). This analysis showed a marginal effect for group $[F(1,8)=$ $4.64, M S_{\mathrm{e}}=.43, p<.061$, with the experts $(M=.62)$ showing better recall than the novices $(M=.46)$. As well, serial position differences were observed $[F(7,56)=$ $\left.14.64, M S_{\mathrm{e}}=.12, p<.001\right]$, with the subjects again showing strong primacy effects $(\mathrm{M} 1=.78, \mathrm{M} 2=.75$, $\mathrm{M} 3=.66, \mathrm{M} 4=.69)$ and no recency effects $(\mathrm{M} 5=$ $.46, \mathrm{M} 6=.36, \mathrm{M} 7=.32, \mathrm{M} 8=.31$ ).

Most important, there were no statistical differences observed for the recall conditions. Although there appeared to be a slight reduction in recall performance for the novices in the two filled-delay conditions and for the experts in the motor-delay condition, these trends were not reliable. Table 1 reflects the performance shown by both groups of subjects for the interpolated-task conditions.

The total-number-of-errors data were analyzed for frequency across conditions. The analysis-a group (expert, novice) and recall condition (immediate, 30 unfilled, 30 count, 30 skate) ANOVA-revealed no significant effect for recall condition, again illustrating the equivalence of performance across conditions.

Since the need to perform an interpolated task between encoding and recall of a figure-skating sequence produced no decrement in recall for either expert or novice skaters, it seems safe to conclude that skaters are not utilizing limited-capacity short-term memory for their encoding. Like chess players and other experts in skilled memory, skaters are using long-term memory.

\section{EXPERIMENT 4}

Figure-skating experts have been shown to be slightly more accurate than their less skilled counterparts in the recall of choreographed skating sequences, although both groups of skaters seem similar in terms of serial-position accuracy (all primacy) and in the use of long-term memory to encode sequences. This is unlike other skilledmemory studies, in which unskilled individuals are quite unable to come close to the experts' level of recall (e.g., Ericsson \& Polson, 1988). One important difference between this investigation and the others is that, although the novice skaters are less skilled, they are not necessarily less experienced than the expert skaters. It is perhaps surprising that the differences between these groups exist at all in the cognitive domain; differences might well be confined to the precision with which skill elements can be executed. In Experiment 4, we continued to explore the cognitive difference between expert and novice skaters in a task involving the learning of a choreographed sequence. In Experiment 4, we hoped to determine whether expert skaters have any particular strategies, such as the retrieval structures described in the skilled-memory literature, or whether skilled skaters are simply better at encoding. The question being asked in Experiment 4 was whether expert and novice skaters differ in degree of encoding or kind of encoding.

The paradigm chosen to evaluate the figure skaters' performance was developed by Chase and Simon (1973a, $1973 \mathrm{~b}$ ) in order to study chunk size in the recall of chess positions. It was anticipated that the use of this paradigm would provide information as to how the individual items were being encoded by figure skaters of varying ability. It was expected that the expert performers would recall more elements per look and would require fewer looks to learn each sequence than would the novice skaters. It was also expected that a pattern of recall performance may appear for the expert performers that might reflect a common analysis of the sequence.

\section{Method}

Subjects. The subjects consisted of 10 skaters -5 experts $(3 \mathrm{fe}-$ males, 2 males), who were members of the Canadian National Team (mean age $=17$ years), and 5 novices (5 females), who were senior bronze and junior silver test free skaters (mean age $=15$ years).

Materials. Stimuli were five free-skating choreographed sequences of eight elements in length. Each sequence was written on a $3 \times 5$ in. card and was made up of elements chosen from a pool of 20 items, with all items being in the repertoire of the novice skaters.

Procedure. The subjects were presented with a card containing an eight-element sequence and allowed to study the sequence for $30 \mathrm{sec}$ ( $4 \mathrm{sec}$ per element). They were instructed to perform the sequence in the order indicated on the card following the study session. The subjects were informed that they could have as many 30 -sec study sessions as needed to produce the sequence perfectly. Following each 30 -sec look, the skater was required to perform the entire sequence from the beginning. Each subject learned all five sequences, with all trials recorded on videotape for later analysis.

\section{Results and Discussion}

The number of elements performed correctly following each study session is shown in Table 2. Clearly, the expert skaters were able to acquire the sequence faster than were the novice subjects, with the difference being greatest over the first two study sessions. To determine 
Table 2

Cumulative Percent Correct for Each Study Session

\begin{tabular}{lccccc}
\hline & \multicolumn{5}{c}{ Study Session } \\
\cline { 2 - 6 } Skater & 1 & 2 & 3 & 4 & 5 \\
\hline Expert & 46.5 & 73 & 92.5 & 98.5 & 99.5 \\
Novice & 31.5 & 62 & 82.5 & 92.5 & 99.0 \\
\hline
\end{tabular}

the nature of the expert-novice difference for initial learning, the first study session was examined in greater detail.

In terms of the number of elements performed correctly following the first 30-sec study session, the experts $(M=3.72)$ were significantly better than the novices $(M=2.52)\left[F(1,8)=6.47, M S_{\mathrm{e}}=.52, p=.03\right]$. Serialposition accuracy for the two groups of subjects following the first study session showed a significant difference for group $\left[F(1,8)=5.2, M S_{\mathrm{e}}=.07, p=.05\right]$ and for serial position $\left[F(7,56)=50.3, M S_{\mathrm{e}}=.028, p=.001\right]$. As well, the interaction between group and serial position was significant $\left[F(7,56)=2.2, M S_{\mathrm{e}}=.028, p=\right.$ $.05]$. This interaction is shown in Figure 3. Tukey WSD post hoc comparisons showed that experts were better for the first four serial positions than the novices, with no group differences occurring for the last four positions because both groups performed so poorly.

From these data, it seems clear that learning for both groups consists of an orderly buildup of the elements in the sequence from first to last items. The serial-position data from Study Session 1 shows that both groups attempted to encode the first four elements following the initial exposure to the sequence. The expert skaters thus differed from the novice skaters in degree of encoding (i.e., they were able to do more in the same time) rather than in kind of encoding.

\section{EXPERIMENT 5}

The experiments presented that show expert skaters to be more accurate at sequence encoding have all involved choreographed skating sequences. This indicates that expert skaters are better than novices because they are better able to take advantage of the sequential dependencies

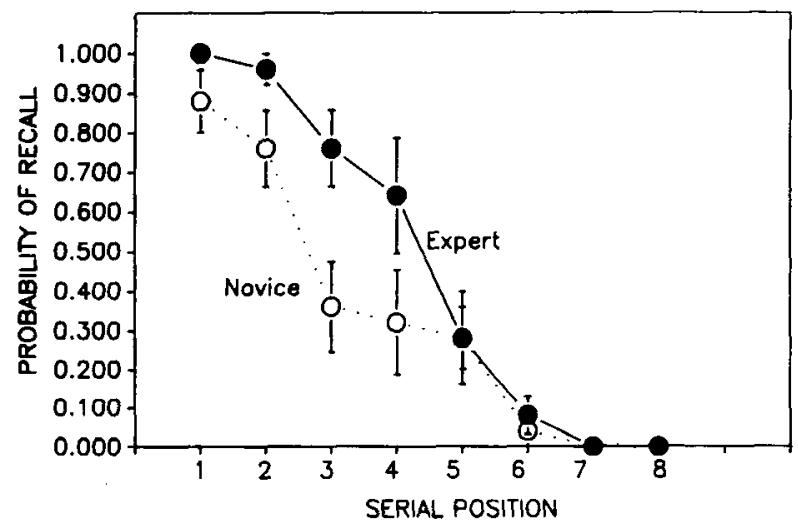

Figure 3. Skill level $\times$ serial position interaction for Look One of Experiment 4. found for choreographed performances, in much the same way as an English-speaking subject is able to take advantage of approximations to English in a recall task (e.g., Miller \& Selfridge, 1950).

There are other factors that well might influence the experts' recall superiority. Performance factors are one possibility. All the elements used in the experiments were easy for the experts, relative to their normal routines. If the experts were using less capacity to perform, more capacity would be available for encoding or rehearsal. Conflicting with this explanation is the finding in Experiment 1 that experts, rather than novices, improve their performance with verbal recall. For all the studies reported in this paper, however, there is little doubt that the performance demands for the novice subjects are much greater than they are for the experts.

As well, experts may be superior at the encoding of individual items, at translating from the verbal representation they were given in this series of experiments to an action representation. Were experts to be faster at this translation step, performance gains likely would accrue.

In Experiment 5, we eliminated the need to perform and the need to translate from verbal to action representation. Expert and novice skaters were compared in their ability to do a simple recognition task, requiring them to judge the similarity of two skating elements. In a task adapted from Posner's (1969) physical/name letter-matching task, skaters observed individual skating elements being performed by the same skater or by two different skaters. The element being observed could be the same or a different element. The task for the skater was to report as rapidly as possible whether the elements were the same or different. Should experts be faster than novices at this task, or should experts show less of a difference between physical and name matches than novices, clear support would be seen for expert skaters' faster access to semantic coding that is independent of sequential dependencies or motor-performance factors. This faster semantic access might well be the basis for the expert skaters' ability to perform more elements correctly than novices following a brief study session of a skating sequence.

Note that the skating same-different task is not identical to the same-different letter-matching task developed by Posner (1969) and widely used in cognitive psychology. In the letter-matching task, trials can be physical matches $(A, A)$, name matches $(A, a)$, or not match $(A, C)$. For the skating task, there are four categories of trials, same skater-same element (analogous to a physical match), different skater-same element (analogous to name match), same skater-different element, and different skater-different element. Thus, there are two categories of different responses in the skating same-different task.

\section{Method}

Subjects. Sixteen figure skaters served as subjects in Experiment 5-8 expert skaters, who were members of the Canadian National Figure Skating Team (mean age $=17.38$ years), and 8 novice skaters, who were senior bronze and junior silver test club skaters. All of the skaters were right-handed. 
Materials. Stimuli consisted of 80 experimental trials of two skating elements performed by a single skater. For half of the trials, the same skater performed both of the elements; and for the other half of the trials, 2 different skaters performed the elements. On half of the trials, the same element was performed twice and, on the other half, different elements were shown. The stimuli were created by editing videotape of international skaters into 20 sameand 20 different-skater trials. The trial order within each block of 20 trials was rerandomized and retaped to provide an additional 40 trials. Trials were thus presented in blocks of 20 , according to whether the same skater or a different skater was performing, for a total of 80 trials.

The stimulus tape was played on a VHS video recorder and displayed on a 14-in. color monitor. A timer was initiated by a marker on the videotape corresponding to the beginning of the second element. Each element was presented for $1,500 \mathrm{msec}$. The subject responded by pressing one of two telegraph keys, with each key triggering an LED indicator on a control box so the experimenter could determine which response had been made.

Procedure. Each subject was tested individually in one 20 -min session. The subjects were seated in front of the monitor and instructed to place their index fingers on the telegraph keys. The subjects were instructed to respond as quickly as possible by pressing the right telegraph key when the elements performed were the same and by pressing the left key when the elements were different. The subjects were also informed as to the nature of the trial block, whether they would be viewing the same skater or different skaters performing the two elements. Time and type of response were recorded by the experimenter.

\section{Results and Discussion}

Data for correct responses for same and different trials were analyzed separately since responding hand was not counterbalanced across subjects. Both data sets were analyzed by means of a group (expert/novice) $\times$ condition (same skater, different skater) ANOVA

For same responses, a main effect was observed for condition $\left[F(1,14)=5.29, M S_{\mathrm{e}}=8,273.48, p=.035\right]$, with responses being faster $(M=892 \mathrm{msec})$ in the sameskater condition than in the different-skater condition $(M=1,028 \mathrm{msec})$. As well, the group $\times$ condition interaction was significant $\left[F(1,14)=5.67, M S_{\mathrm{e}}=8,273.48\right.$, $p=.03]$. Tukey WSD post hoc comparisons for the interaction showed that the experts did not differ for responses to same and different skaters, whereas the novices were slower for the different-skater condition than they were for the same-skater condition. As well, the novices were slower than were the experts for differentskater judgments. The condition means are shown in Table 3. For different responses, a marginally significant effect for group was observed $\left[F(1,14)=3.65, M S_{\mathrm{e}}=\right.$ $90,001.98, p=.074]$, with the experts responding faster than did the novices. As well, same-skater judgments were significantly faster than different-skater judgments $\left[F(1,14)=27.21, M S_{\mathrm{e}}=5,451.69, p<.001\right]$ (see Table 3).

In light of the small number of errors committed, the error data were collapsed across trial type. This analysis showed a significant main effect for group $[F(1,14)=$ $10.06, M S_{c}=4.03, p=.006$ ], with the experts making fewer errors than did the novices. All subjects were more accurate for same-skater trials $\left[F(1,14)=56.26, M S_{\mathrm{e}}=\right.$
Table 3

Mean Response Times (in milliseconds) and Error Rates for Same-Different Task

\begin{tabular}{lrcrrr} 
& \multicolumn{2}{c}{ Same Skaters } & & \multicolumn{2}{c}{ Different Skaters } \\
\cline { 2 - 3 } \cline { 5 - 5 } Skater & Mean RT & Error Rate & & Mean RT & Error Rate \\
\hline Expert & 900.07 & 203.46 & & 897.53 & 209.88 \\
Novice & $1,013.28$ & 323.95 & & $1,162.83$ & 340.86 \\
& \multicolumn{5}{c}{ Different Responses } \\
Expert & 813.23 & 195.95 & 904.36 & 168.80 \\
Novice & 970.87 & 220.86 & & $1,152.08$ & 274.28 \\
& \multicolumn{5}{c}{ Same Responses } \\
Expert & 1.00 & Errors & & \\
Novice & 1.25 & 2.75 & 3.12 & 1.45 \\
\hline
\end{tabular}

$2.42, p<.0011$. Finally, the group $\times$ condition interaction was significant $\left[F(1,14)=13.23, M S_{\mathrm{e}}=2.42\right.$, $p=.002$ ]. Tukey WSD post hoc comparisons showed that the experts and the novices did not differ in error rate for same-skater judgments; however, the experts made fewer errors than did the novices for different-skater judgments and more errors than for their own same-skater judgments, as shown in Table 3.

There was no intention in the choice of the present experimental task to interpret the resulting data in terms of physical and name codes. Clearly, the task of the skater is to compare two elements that unfold over time rather than to compare two patterns that are simultaneously present. It remains for future work to determine the nature of the representation used by closed-skill athletes, such as skaters, to encode skill elements.

The case we wish to make here is purely descriptive: Expert skaters are able to determine that two skating elements are identical when they are performed by different skaters in the same amount of time as when the elements are performed by the same skater, whereas novice skaters are slowed significantly by the need to do a cross-skater comparison. This finding is confirmed by the errors made in comparisons: Although both groups made more errors in different-skater comparisons, the novices' error rate was significantly higher than was their own error rate for same-skater comparisons and for experts' different-skater comparisons. The expert and the novice skaters were faster at deciding that two elements were different when the elements were performed by the same skater, but the experts were faster overall than the novices in these judgments. As Table 3 shows, the response times of the expert subjects are remarkably stable across the various conditions and are about $200 \mathrm{msec}$ faster than the response times of the novices. It thus appears that for a task involving no actual performance of skating elements and no translation from semantic memory to action, expert skaters still show a superiority over novice subjects. Should expert skaters have faster access to the representations of skating elements, it is logical that they would be able to perform more elements correctly following the study of a skating sequence. 


\section{GENERAL DISCUSSION}

In this series of five experiments, we have shown the following: (1) Expert figure skaters are able to recall more elements of a figure-skating sequence when the sequence is choreographed. This finding provides evidence for a domain-specific encoding ability in the skaters. A comparison of serial-position accuracy for lists of skating terms coded for verbal report and that coded for performance shows very different patterns. Thus, figure skaters do not simply memorize to-be-performed sequences in the same fashion as they memorize verbal lists. (2) Long-term memory is implicated in the learning of skating sequences for both novice and expert skaters, since both groups were little influenced by tasks interpolated between learning and performing a skating sequence. (3) As do experts in other skilled-memory domains, skating experts are able to recall more elements following a brief study session, but (4) unlike other experts, this does not seem to be the product of mnemonic coding or retrieval structures. (5) Instead, expert skaters seem to have faster access to semantic memory for skating elements than do novices, as shown by their ability to perform the simple task of judging the similarity of two skating elements faster than can novices.

Although we have been unable to show that skaters use retrieval structures in the performance of the short sequences used in the course of these experiments, it should be pointed out that one aspect of the typical skating environment was missing. A figure-skating routine is always performed with music, which could act as a powerful mnemonic aid. In fact, Starkes et al. (1987) have shown that the recall performance of young ballet experts is aided by having music present for both encoding and recall of ballet sequences. As well, the eight-item sequences that our subjects had to recall might not tax individuals who must learn performance routines lasting for many minutes and consisting of hundreds of individual elements as we have defined them. It appears that athletes in a domain that places minimal demands on cognition and maximal demands on precision of execution show many of the same memory skills that characterize the performance of experts in such domains as digit span (Chase \& Ericsson, 1982) and recall of dinner orders (Ericsson \& Polson, 1988).

\section{REFERENCES}

allard, F., Graham, S., \& Paarsalu, M. (1980). Perception in sport: Basketball. Journal of Sport Psychology, 2, 14-21.
Charness, N. (1976). Memory for chess positions: Resistance to interference. Journal of Experimental Psychology: Human Leaming \& Memory, 2, 641-653.

Charness, N. (1979). Components of skill in bridge. Canadian Journal of Psychology, 33, 1-16

Chase, W. G., \& Ericsson, K. A. (1982). Skill and working memory. In G. Bower (Ed.), The psychology of learning and motivation (Vol. 16, pp. 1-58). New York: Academic Press.

Chase, W. G., \& Simon, H. (1973a). Perception in chess. Cognitive Psychology, 4, 55-81.

Chase, W. G., \& Simon, H. (1973b). The mind's eye in chess. In W. G. Chase (Ed.), Visual information processing (pp. 215-281). New York: Academic Press.

ChI, M. T. H., Feltovich, P. J., \& Glaser, R. (1981). Categorization and representation of physics problems by experts and novices. Cognitive Science, 5, 121-152.

ChI, M. T. H., Glaser, R., \& FARR, M. J. (Eds.) (1988). The nature of expertise. Hillsdale, NJ: Erlbaum.

Ericsson, K. A., Chase, W. G., \& Faloon, S. (1980). Acquisition of a memory skill. Science, 208, 1181-1182.

Ericsson, K. A., \& Polson, P. G. (1988). A cognitive analysis of exceptional memory for restaurant orders. In M. T. H. Chi, R. Glaser, \& M. J. Farr (Eds.), The nature of expertise (pp. 23-70). Hillsdale, NJ: Erlbaum.

FreY, P. W., Adesman, P. (1976). Recall memory of visually presented chess positions. Memory \& Cognition, 4, 541-547.

Groen, G. J., \& PATEL, V. L. (1988). The relationship between comprehension and reasoning in medical expertise. In M. T. H. Chi, R. Glaser, \& M. J. Fart (Eds.), The nature of expertise (pp. 287310). Hillsdale, NJ: Erlbaum.

Miller, G. A., \& Selfridge, J. A. (1950). Verbal context and the recall of meaningful material. American Joumal of Psychology, 63, 176-185.

POSNER, M. I. (1969). Abstraction and the process of recognition. In G. H. Bower \& J. T. Spence (Eds.), The psychology of learning and motivation: Advances in research and theory (Vol. 3, pp. 43-100). New York: Academic Press.

Rertman, J. S. (1976). Skilled perception in Go: Deducing memory structures from inter-response times. Cognitive Psychology, 8, 336-356

Soloway, E., Adelson, B., Ehrlich, K. (1988). Knowledge and processes in the comprehension of computer programs. In M. T. H. Chi, R. Glaser, \& M. J. Farr (Eds.), The nature of experiise (pp. 129-152). Hillsdale, NJ: Erlbaum.

Starkes, J. L., \& Deakin, J. M. (1984). Perception in sport: A cognitive approach to skilled performance. In W. F. Straub \& J. M. Williams (Eds.), Cognitive sport psychology (pp. 115-128). Lansing, NY: Sport Science Associates.

Starkes, J. L., Deakin, J. M., Lindley, S., \& Crisp, F. (1987). Motor versus verbal recall of ballet sequences by young expert dancers. Journal of Sport Psychology, 9, 222-230.

STASZEWSKI, J. J. (1988). Skilled memory and expert mental calculation. In M. T. H. Chi, R. Glaser, \& M. J. Farr (Eds.), The nature of expertise (pp. 71-128). Hillsdale, NJ: Erlbaum.

Zacks, R. T., Hasher, L., AlaA, J. W., SANFt, H., Rose, K. C. (1984). Is temporal order encoded automatically? Memory \& Cognition, 12, 387-394.

(Manuscript received June 21, 1989; revision accepted for publication May 30, 1990.) 